\title{
The Art of Remediating Age-Related Cognitive Decline: Art Therapy Enhances Cognition and Increases Cortical Thickness in Mild Cognitive Impairment
}

\author{
Junhong $\mathrm{Yu}^{1, *}$ (D) , Iris Rawtaer ${ }^{2}$, Lee Gan $\mathrm{Goh}^{3}$, Alan Prem Kumar ${ }^{4,5}$, Lei Feng ${ }^{1}$, Ee Heok Kua ${ }^{1}$ and Rathi Mahendran ${ }^{1,6, *}$ \\ ${ }^{1}$ Department of Psychological Medicine, Mind Science Centre, Yong Loo Lin School of Medicine, National University of Singapore, \\ 119077 Singapore, Singapore \\ ${ }^{2}$ Department of Psychiatry, Sengkang General Hospital, 544886 Singapore, Singapore \\ ${ }^{3}$ Department of Family Medicine, National University Health System, 119228 Singapore, Singapore \\ ${ }^{4}$ Department of Pharmacology, Yong Loo Lin School of Medicine, National University of Singapore, 117600 Singapore, Singapore \\ ${ }^{5}$ Cancer Science Institute of Singapore, National University of Singapore, 117599 Singapore, Singapore \\ ${ }^{6}$ Academic Development Department, Duke-NUS Medical School, 169857 Singapore, Singapore
}

(Received March 1, 2020; Final revision May 6, 2020; Accepted June 8, 2020; First Published Online August 7, 2020)

\begin{abstract}
Objective: Previous research on art therapy (AT) in cognitive aging has been lacking. AT can potentially engender significant cognitive gains, due to its rigorous cognitive involvement, making it useful to tackle age-related cognitive decline. Along with these cognitive gains, associated neuroplastic changes are hypothesized to arise from AT as well. The current intervention examined the effects of an AT intervention on cognitive outcomes and cortical thickness (CT) among participants with mild cognitive impairment. Method: Participants were assigned to AT $(n=22)$ and an active control group $(n=27)$. In both, weekly 45 -min sessions were carried out across 3 months. Cognitive assessments and structural magnetic resonance imaging scans were carried out at baseline and 3-month follow-up. Whole brain analyses on CT were carried out. Cognitive outcomes were analyzed using hierarchical linear models. Results: Significant gains in immediate memory and working memory span were observed in the AT group, relative to the control group. Significantly increased CT in the AT group, relative to controls, was observed in a right middle frontal gyrus (MFG) cluster. Furthermore, CT changes in this cluster were significantly and positively correlated with changes in immediate memory. Conclusion: These findings highlighted the role of MFG neuroplasticity in enhancing certain cognitive functions in AT. AT is a neuroplastic intervention capable of engendering significant cognitive gains and associated cortical changes in the context of age-related cognitive decline, even when executed as a low-intensity intervention across 3 months. Given the preliminary nature of these findings, future larger sampled studies are needed.
\end{abstract}

Keywords: Art therapy, Cognitive enhancement, Memory, Cortical thickness, Neuroplasticity, Mild cognitive impairment

\section{INTRODUCTION}

The mild cognitive impairment (MCI) diagnostic entity was conceptualized to describe the intermediate stage between normal aging and dementia, drawing attention to the importance of intervening at this stage to alter the course of subsequent cognitive decline (Petersen et al., 2014).

*Correspondence and reprint requests to: Dr. Yu Junhong, Research Fellow, Department of Psychological Medicine, Yong Loo Lin School of Medicine, National University of Singapore, MD1 Research Dry Laboratories (North), \#12-01, 12 Science Drive 2, 117549, Singapore, E-mail: pcmyj@nus.edu.sg; Dr. Rathi Mahendran, Senior Consultant and Associate Professor, Department of Psychological Medicine, Yong Loo Lin School of Medicine, National University of Singapore, NUHS Tower Block, Level 9, 1E Kent Ridge Road, 119228, Singapore, Tel: +(65) 67723893, Fax: +(65)67772191, E-mail: pcmrathi@nus.edu.sg
Non-pharmacological interventions for MCI typically involve cognitive training or physical activity (Rodakowski, Saghafi, Butters, \& Skidmore, 2015), which often require effortful and repetitive training/execution of various tasks. While such intervention reportedly produced reliable cognitive gains (Sherman, Mauser, Nuno, \& Sherzai, 2017) and can be efficiency administered to large samples via computers (Lampit, Hallock, \& Valenzuela, 2014), they have been described as uninteresting to the participants (Till, Kuni, De Somma, Yeh, \& Banwell, 2019; Törnbom \& Danielsson, 2018). Arguably, they are hardly motivating enough for one to be voluntarily engaged on a long-term basis in order to reap sustained cognitive benefits. Furthermore, there is a relatively low limit to how many times a given cognitive training task can be repeated before decreasing marginal returns sets in. Hence, 
there is a need to look at other more interesting and sustainable approaches to enhancing cognitive functioning, in the context of age-related cognitive decline.

Art therapy (AT), the use of art-making activities to enhance well-being and overcome various difficulties and challenges, is one such approach (Ulman, 2001). AT has been typically carried out as a means to facilitate emotional expression and resolve psychoaffective issues (Ulman, 2001). Not surprisingly, most AT interventions have targeted psychiatric outcomes, such as depression and anxiety (Maujean et al., 2014). Nevertheless, AT can be used to enhance cognitive functioning as well. In particular, AT, via its art production and evaluation components, trains upon several cognitive processes. For instance, visual art production requires one to visualize various possibilities of the final art piece and iterate between different possibilities. In deciding between possible artistic designs, the artist has to integrate multiple types of information such as those in the visual and emotional domains to arrive at a judgment (Kontson et al., 2015). Then, he has to hold the design in his working memory or short-term memory, while he replicates the design out in real life. In doing so, he may have to plan out several intermediate steps in a goal-directed manner. While executing these steps, he needs to constantly self-monitor to ensure that the artwork is going according to plan and is visually appealing. As for art evaluation, on top of integrating emotional and aestheticrelated information arising from the artwork, one has to engage in perspective-taking to imagine the thoughts and feelings of the artist who created the art piece. Taken together, AT, via its art production and evaluation components, recruits heavily upon executive functions, visuospatial, and sociocognitive processing.

With the long-term cognitive stimulation and engagement from multiple sessions of AT, it is likely that AT could lead to enhanced cognitive functioning. For instance, one intervention assigned older adults to a group-based AT-involving drawing, coloring, collage, and simple crafting activities, or an active control (AC) group, and observed significant improvements in the clock drawing test (i.e., visuospatial processing) in the former group (Pike, 2013). However, two other AT randomized controlled trials (RCTs) (Hattori, Hattori, Hokao, Mizushima, \& Mase, 2011; Rusted, Sheppard, \& Waller, 2006) on patients with dementia have failed to obtain significant AT-related cognitive gains, as measured via the mini-mental state examination (MMSE) and logical memory from the Wechsler Memory Scale. Perhaps, the MMSE, which measures global cognition, may not be sensitive enough to detect AT-related treatment gains on certain specific cognitive domains. Furthermore, it is possible that AT, due to the nature of its cognitive involvements, is unlikely to result in memory improvements, relative to those of executive functions and visuospatial processing.

These AT-associated cognitive gains are likely to be accompanied by experience-dependent neuroplastic changes, especially in the prefrontal cortex (PFC) - a region that was theorized to be heavily involved in AT (Lusebrink, 2004). Although a previous study has identified AT-associated functional changes in the brain, especially in the default mode network (Bolwerk, Mack-Andrick, Lang, Dörfler, \& Maihöfner, 2014), the neuroplastic mechanisms of AT remained unstudied thus far.

To these ends, we sought to bolster the evidence on the cognitive gains and uncover the neuroplastic mechanisms of AT. The current study analyzed a community sample of older adults with MCI who were assigned to an AT intervention or an appropriate control treatment. We administered a battery of neurocognitive tests to assess a variety of cognitive domains and acquired structural magnetic resonance imaging (MRI) scans to examine the changes in gray matter (GM) cortical thickness (CT). In line with previous research, we hypothesized that AT would lead to significant cognitive gains, especially in the domains of executive functions and visuospatial processing, and increased $\mathrm{CT}$ in the prefrontal regions.

A previous study (R. Lee et al., 2019) had analyzed the age- and education-normed cognitive scores of the same group of AT participants together with a no-contact control group, across a 9-month intervention period. Despite using the same group of AT participants, the current study makes three additional contributions. First, we paired AT with an $\mathrm{AC}$ group so as to justify stronger interpretations. It should also be noted that the psychiatric and MMSE scores of this AC group were analyzed previously (Klainin-Yobas et al., 2019), in comparison to a mindfulness meditation group. Second, we analyzed raw cognitive scores, as opposed to normed scores; due to certain artifacts in the norming process, normed scores may not be sensitive in tracking longitudinal changes. Third, the current study analyzed structural MRI data in the form of GM CT; no such structural MRI data, of any form, were analyzed previously. Our analyses were restricted to the first 3 months, since MRI data were only acquired at baseline and 3-month follow-up for the AT sample, and the frequency of sessions in AT and AC differed after the first 3 months. Taken together, although we used a previously analyzed sample of AT participants in the present study, there were no overlaps in the analyzed interventionrelated outcomes in the present and previous studies.

\section{METHOD}

\section{Design}

Data from the current study came from two open-label RCTs (Clinical Trial Registry Numbers: NCT02854085 and NCT02286791). The first randomized eligible participants to one of three groups-AT, Music Reminiscence Activity and a no-contact control group (R. Lee et al., 2019), and the second randomized participants to a mindfulness awareness program or an AC group (Klainin-Yobas et al., 2019). Both RCTs had similar assessment and recruitment protocols. For the current study, we examined the effects of AT in the first RCT, with the AC in the second RCT used as a control. We did not use the no-contact control group within the RCT that included the AT due to two reasons. First, unlike the AC which was carried out in a 


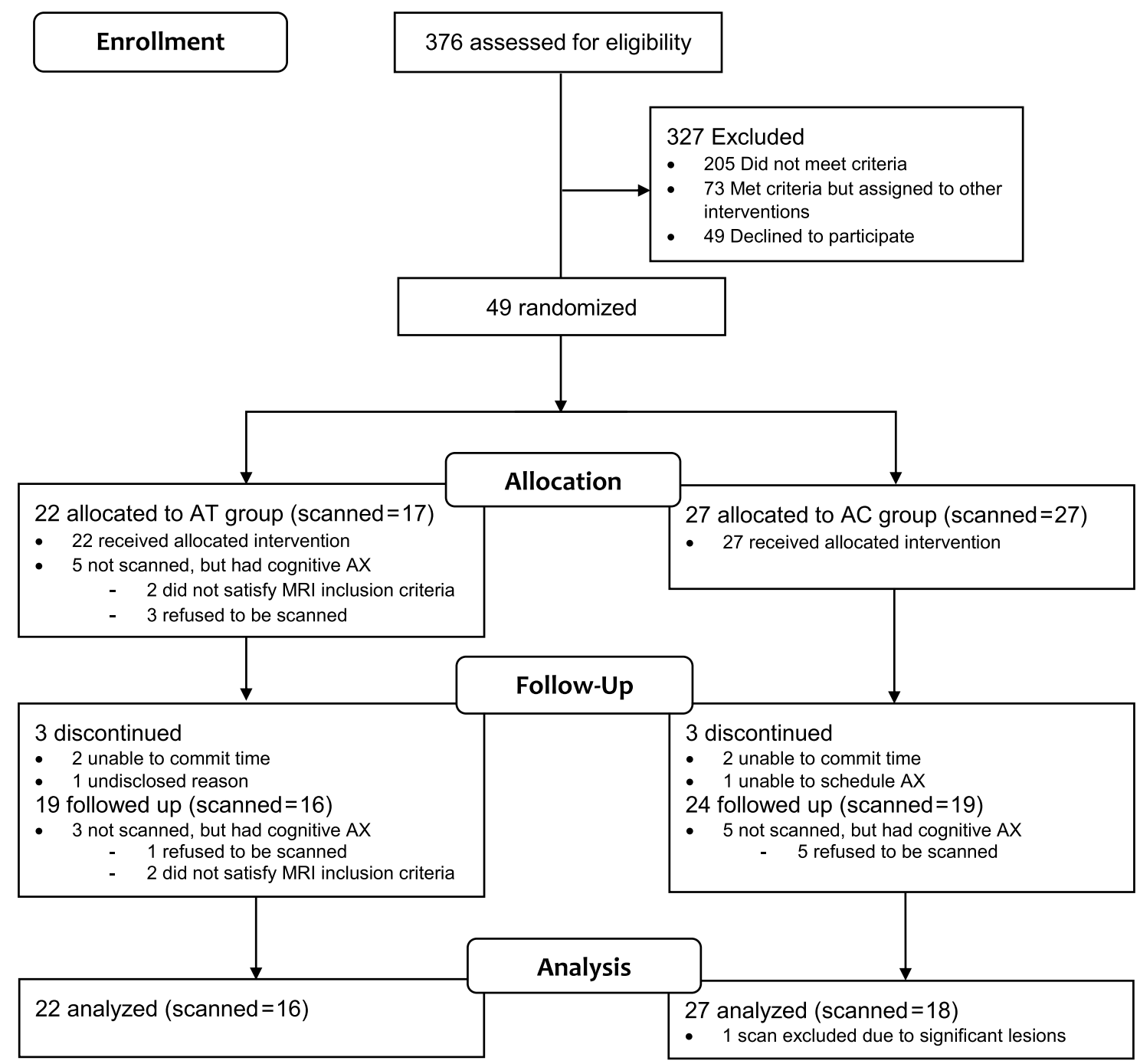

Fig. 1. Consort flow diagram. $\mathrm{MCI}=$ mild cognitive impairment; $\mathrm{AT}=\mathrm{Art}$ Therapy $\mathrm{AC}=\mathrm{Active}$ control; $\mathrm{AX}=$ assessment.

group-based setting, the no-contact control group does not provide opportunities for social interactions. Given that our AT was carried out in a group-based setting thus facilitating social interactions, the AC would be necessary to control for the effects of social interaction. Secondly, the number of scanned participants in the no-contact control group was small $(N=10)$. In both the AT and AC groups, the intervention spanned across 3 months. Twelve weekly sessions were held across this period, each lasted approximately $45 \mathrm{~min}$. The CONSORT flow diagram of the study is shown in Figure 1. Ethical approval for the current study was granted by the National University of Singapore Institutional Review Board.

\section{Participants}

Participants of the current study were recruited from the community. The inclusion criteria were (1) aged between 60 and 85 (inclusive), (2) met the criteria for MCI, (3) able to travel to the intervention venue independently, (4) not presenting with any current psychiatric or neurological conditions, (5) not suffering from any terminal illness, (6) not participating in another interventional study and for those undergoing MRI scans, and (7) not having any metallic body implants (e.g., pacemaker), with the exception of dental implants.

The Petersen's (2004) criteria were used for the MCI diagnosis. These include subjective cognitive complaints as corroborated by a reliable informant, presence of objective cognitive impairment and largely preserved functional independence. In particular, the objective cognitive impairment criterion was operationalized as scoring 1.5 standard deviations (SDs) below the age and education appropriate norms in any of five cognitive domains, as assessed via the digit span and block design tests from Wechsler Adult Intelligence Scale (WAIS-III), delayed recall and recognition tests from the Rey Auditory Verbal Learning Test (RAVLT), and Color Trails Test (CTT). The MCI diagnoses were made via a consensus from a panel of psychiatrists and psychologists.

In total, 22 were assigned to the AT group and 27 to the AC control group. Written informed consent was obtained 
Table 1. Characteristics of participants assigned to both treatment conditions

\begin{tabular}{|c|c|c|c|c|c|}
\hline & \multirow[b]{2}{*}{$\operatorname{AT}(N=22)$} & \multirow[b]{2}{*}{$\mathrm{AC}(N=27)$} & \multicolumn{3}{|c|}{ Between-group comparison } \\
\hline & & & $t$ & $\chi^{2}$ & $p$ \\
\hline Mean age (SD) & $71.14(4.95)$ & $71.44(5.97)$ & 0.14 & & 0.89 \\
\hline \multicolumn{6}{|l|}{ Sex } \\
\hline Males & 4 & 5 & & 0.50 & 0.82 \\
\hline Females & 18 & 19 & & & \\
\hline Mean years of education (SD) & $5.17(4.27)$ & $3.44(4.27)$ & 0.96 & & 0.34 \\
\hline Mean MMSE score (SD) & $25.06(3.04)$ & $24.70(3.89)$ & 0.12 & & 0.90 \\
\hline Mean sessions attended ${ }^{\mathrm{a}}(\mathrm{SD})$ & $10.68(1.73)$ & $10.75(1.36)$ & 0.15 & & 0.88 \\
\hline \multicolumn{6}{|l|}{ Language of assessment } \\
\hline Mandarin & 19 & 21 & & 5.99 & 0.20 \\
\hline Hokkien & 0 & 4 & & & \\
\hline Cantonese & 0 & 1 & & & \\
\hline Teochew & 1 & 0 & & & \\
\hline English & 2 & 1 & & & \\
\hline
\end{tabular}

${ }^{\mathrm{a} A m o n g}$ the analyzed longitudinal sample.

prior to participation. Forty-three participants from both groups completed their respective interventions, and their mean attendance was at $10.72(\mathrm{SD}=1.52)$ sessions. These participants included three from the AT group who did not complete the second part of the CTT during the baseline time point, hence they did not have valid CTT-interference scores. Both groups were not significantly different in demographics, MMSE scores, and attendance ( $p$ s $\geq .34$; see Table 1). As observed from the low average number of years of education in both groups, most participants did not complete their primary school (local equivalent of elementary school) education, which consisted of 6 years of schooling.

Although we intended to analyze the treatment effects using hierarchical linear models (HLMs), the sample size calculations for such statistical models are complicated. As such, we determined the minimum sample size to detect a withinbetween interaction effect in a repeated measure analysis of variance model, to approximate the sample size required to detect treatment effects in our HLM analyses. Our calculations using $\mathrm{G}^{*}$ Power indicated that the current sample of completers would provide a power of .80 (assuming an alpha level of .05 and a .5 correlation between repeated measures) to detect a significant Time $\times$ Group interaction if the true effect was at least $d=.44$. Given that a meta-analysis of cognitive training interventions on the MCI population reported a Hedge's G (comparable to Cohen's d) of .454 (Sherman et al., 2017), we are confident that the current sample size is more than adequate to detect the hypothesized cognitive gains.

\section{Measures}

The Digit Span subtests from the WAIS-III (Wechsler, 1997) were used to index the participant's working memory. These tests require participants to immediately recall a series of numbers after they were read out, in the order that they were read out (forward) or in a reversed order (backward). The forward trial measures auditory attention span, whereas the backward trial measures the ability to manipulate information in working memory. The total scores, as opposed to span scores, within each variant of the test were used.

The CTT (D'Elia \& Satz, 1996) was used to assess divided attention. The CTT consists of two parts. In the first, participants connected a series of numbers that were printed within colored circles, sequentially from 1 to 25 . Subsequently, in the second part, participants alternated between numbers in either pink or yellow circles, while similarly connecting the numbers from 1 to 25 . Divided attention was operationalized as the interference effect, which is the difference in completion times of the first and second parts, divided by the former.

The RAVLT (Rey, 1941) was used to assess immediate and delayed verbal memory, as well as recognition memory. Participants were given a list of 15 unrelated words (list A) to learn and immediately recall aloud over five learning trials (Total learning). Subsequently, an interference list of 15 unrelated words (list B) was presented only once for the participants to learn and recall immediately. Following this, the participants were instructed to recall aloud the words from list A. Approximately $30 \mathrm{~min}$ later, they were again asked to recall aloud the words from list A (Delayed Recall). Finally, they were given a list of 50 words, comprising of list A, list B, and 20 new distractor words, from which they had to identify the original 15 words (Recognition).

The block design test from the WAIS-III was used to assess visuospatial functions. In this test, the participant was instructed to arrange blocks with red and white patterns on different sides, in a manner to replicate the required block patterns in each trial. Scoring for the block design test depended not only on the accuracy of replicating the block patterns but speed as well; participants were awarded extra points for completing the trials within various time limits.

These tests were administered in English, Mandarin, and other Chinese dialects according to the participants' 
dominant language. These non-English translations have been described in a previous study (Lee, Collinson, Feng, \& $\mathrm{Ng}, 2012$ ).

\section{Intervention Protocol}

The AT was developed by our research team in consultation with art therapists. This 12-week AT intervention consisted of six 'art-making and sharing' sessions interspersed with six art gallery visits. Different themes (e.g., family and friendship) were assigned in each of the 'art-making and sharing' sessions. Participants used a variety of materials, such as paint, pencils, crayons, picture cards, pastels, stickers, and colored paper to create drawings, collage work, symbol work, or clay work/sculptures, in relation to a weekly assigned theme. Then, they shared their thoughts and feelings on their artwork, first in pairs and then within the group. During the group sharing, art therapists guided the participants in appraising their artwork cognitively and emotionally by using probing questions (e.g., "why was this person drawn in this way?")

The art gallery visits took place at the National Art Gallery and the National University of Singapore Art Museum. In these visits, participants gathered around pre-selected art pieces (e.g., paintings, drawings, heritage objects) and were introduced to the artwork by the art therapist. Subsequently, they shared among themselves, their perspectives and feelings on the art piece. During which, the art therapist facilitated the sharing by posing questions on the theme of the artwork (e.g., "If you can give this painting a title, what would it be?"), visual observations (e.g., "Which part of this painting captures your attention?"), feelings (e.g., "What do you feel when you look at this painting?"), and specific details of the artwork (e.g., "Why do you think the character of this painting was drawn this way?").

The AC was executed as didactic sessions on healthy lifestyle and was carried out in a quiet room within a community location. These sessions were taught by an experienced instructor and covered topics such as chronic medical conditions (e.g., hypertension, diabetes, and arthritis), medication compliance, and healthy lifestyles (e.g., diet, nutrition, exercise, and relaxation).

\section{MRI Acquisition}

Participants were scanned using a 3-T Siemens Tim Trio scanner at the Clinical Imaging Research Center, Singapore. T1-weighted images were acquired using an Magnetization Prepared - RApid Gradient Echo (MPRAGE) protocol (echo time $[\mathrm{TE}]=1.90 \mathrm{~s}$; repetition time $[\mathrm{TR}]=2.30 \mathrm{~s} ;$ inversion time $[\mathrm{TI}]=900 \mathrm{~ms} ; 256 \times 256$ matrix; 192 sagittal slices; in-plane resolution $=1 \mathrm{~mm}$; slice thickness $=1 \mathrm{~mm}$ ).

\section{Image Preprocessing}

T1-weighted images were preprocessed longitudinally using the CAT12 (version r1450) as implemented in SPM12 (version 6225) within MATLAB (R2019a). Baseline and follow-up images were rigidly realigned within subject to correct for differences in head position, and a subject-specific average template was computed and used as a reference in a subsequent realignment of the baseline and follow-up images. The realigned images were segmented into GM, white matter (WM), and cerebrospinal fluid, as well as corrected for signal inhomogeneities with reference to the subject average template. These GM and WM images were used to estimate spatial normalization deformation fields using the high-dimensional Diffeomorphic Anatomic Registration Through Exponentiated Lie Algebra warping algorithm (Ashburner, 2007), which were applied to segmented images and subsequently modulated. These modulated images were then visually inspected.

Estimation of CT and the central surface was carried out using the projection-based thickness method (Dahnke, Yotter, \& Gaser, 2013), which consisted of topology correction (Yotter, Dahnke, Thompson, \& Gaser, 2011), spherical mapping, and spherical registration (Yotter, Thompson, \& Gaser, 2011). After obtaining the segmented images, WM distance was estimated and the local maxima were projected to other GM voxels using a neighbor relationship described by the WM distance (Dahnke et al., 2013).

\section{Statistical Analyses}

To assess for AT-related changes in cognitive outcomes, we modeled each outcome using HLMs. The HLM analyses were carried out using the lmerTest package in $\mathrm{R}$ 3.6.1. Statistical significance was set at $p<.05$. This approach was used because we assumed that the missing data occurred at random and we were able to maximize the use of all available data-even the baseline data of those who did not complete the 3-month assessments. No data imputation or replacement were carried out. HLM has been shown to produce unbiased results when there are missing data (Fielding, Fayers, \& Ramsay, 2012). This model consisted of a random intercept $\left(\beta_{\mathrm{i} 0}\right)$ and the fixed effects of group, time and group $\times$ time interaction. The model is specified below:

Level 1:

$$
\begin{aligned}
y_{i j}= & \beta_{i 0}+\beta_{i 1}(\text { group })+\beta_{i 2}(\text { time }) \\
& +\beta_{i 3}(\text { group } \times \text { time })+e_{i j}
\end{aligned}
$$

where $y_{\mathrm{ij}}$ represents the test score for participant $\mathrm{i}_{\mathrm{i}}$ at time $\mathrm{j}_{\mathrm{j}}$ ( $\mathrm{j}$ refers to the baseline or 3 -month time points); $\beta_{\mathrm{i} 0}$ represents the random intercept for participant ${ }_{\mathrm{i}} ; \beta_{\mathrm{i} 1}$ represents the fixed effect of group for participant $; \beta_{\mathrm{i} 2}$ represents the fixed effect of time for participant ${ }_{\mathrm{i}} ; \beta_{\mathrm{i} 3}$ represents the fixed effect of group $\times$ time interaction for participant $\mathrm{i}_{\mathrm{i}}$.

Level 2:

$$
\beta_{i 0}=\gamma_{00}+u_{i 0}
$$

where $\gamma_{00}$ represents the intercept for all participants; $u_{\mathrm{i} 0}$ represents the residual. 

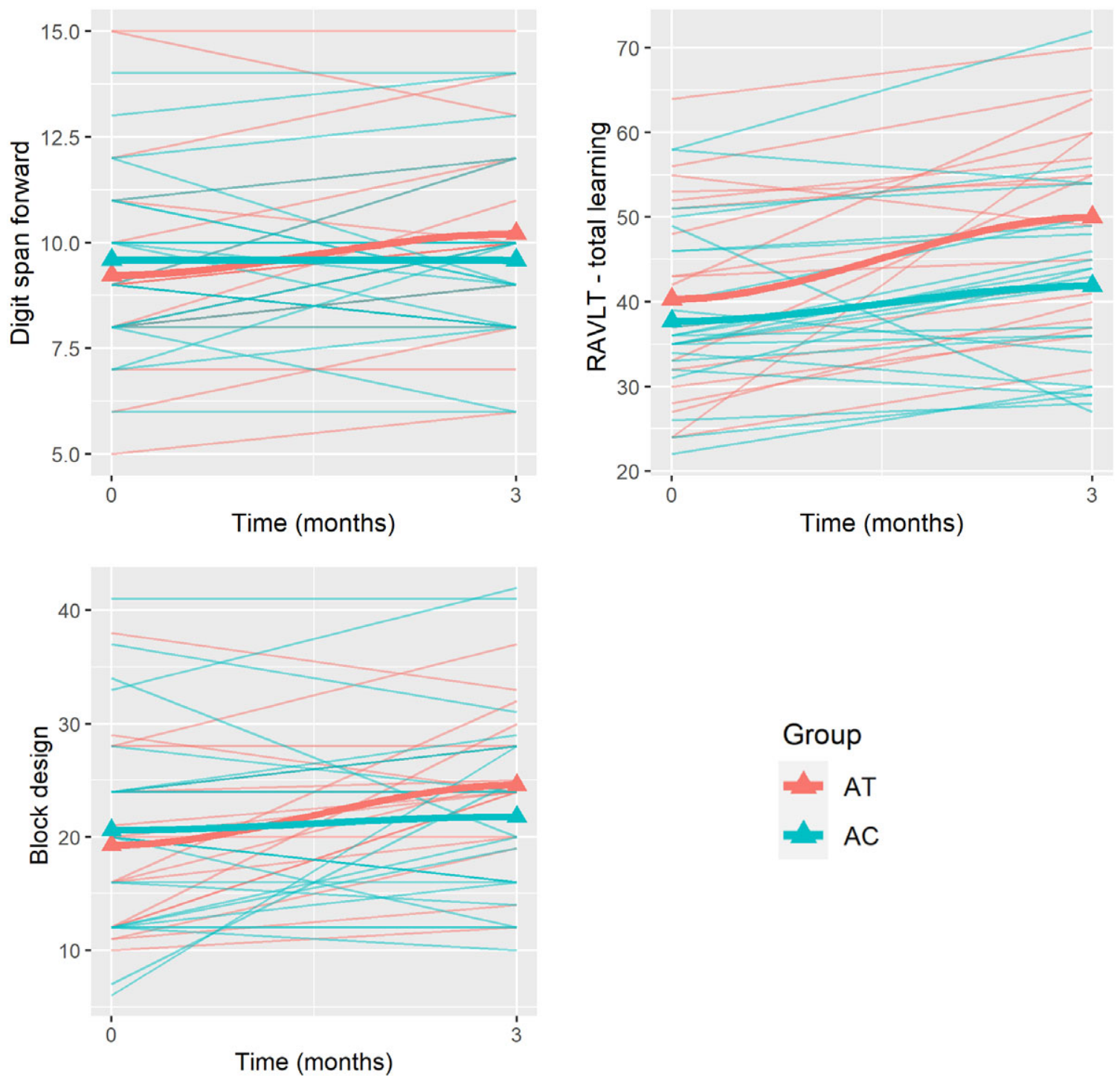

Fig. 2. Trajectories of digit span forward, RAVLT - Total learning and block design scores across the two treatment groups.

Due to the fact that the longitudinal preprocessing pipeline in CAT12 can only be carried out on subjects with both baseline and follow-up scans, unlike the HLM analyses described above, we could not include the baseline scans of subjects who were not scanned at follow-up. Whole brain analyses were carried out using CAT12 on the preprocessed surface images. Prior to the analyses, one subject from the AC group was excluded due to significant GM lesions, possibly caused by brain surgery. Then, treatment effects were assessed using a flexible factorial general linear model incorporating the three factors of Subject, Group, and Time. We examined the treatment effects within the $\mathrm{T}$ contrasts for the Group $\times$ Time interaction (i.e., 3 months $>$ Baseline and $\mathrm{AT}>\mathrm{AC}$ and 3 months $>$ Baseline and AT $<\mathrm{AC}$ ). The results were thresholded using cluster-level family-wise error (FWE) corrected $p<.05$, against a null distribution generated by 5000 nonparametric permutations using the Threshold-Free Cluster Enhancement (TFCE) toolbox, with a cluster extent threshold of $k>20$. Significant clusters are labeled using the Desikan-Killiany atlas (Desikan et al., 2006).

\section{RESULTS}

\section{Effect of AT on Cognitive Outcomes}

The descriptive statistics of all cognitive outcomes across both time points are reported in Supplementary Table 1. There was a significant Time $\times$ Group effect on digit span forward $(\beta=.42,95 \%$ confidence interval $(\mathrm{CI})=[.02, .81]$, $p=.044)$ and RAVLT-Total learning $(\beta=.50,95 \%$ $\mathrm{CI}=[.07, .92], p=.026)$. There was also a marginally significant Time $\times$ Group effect on block design scores $(\beta=.46$, $95 \% \mathrm{CI}=[-.05, .97], p=.081)$. The plotted trajectories of these cognitive outcomes in Figure 2 showed that AT, relative to $\mathrm{AC}$, led to improved outcomes in these tests. The full parameters ( $\beta, \mathrm{df}$, CIs, and $\mathrm{p}$ values) of $\beta_{0}$ to $\beta_{4}$ are presented in Supplementary Table 2.

\section{Effect of AT on CT}

Whole brain analyses within the contrast of 3 months $>$ baseline \& AT $>$ AC revealed a significant cluster in the right 
Table 2. Results of the 3 months $>$ baseline and AT $>$ AC contrast

\begin{tabular}{lccccc}
\hline \hline \multirow{2}{*}{ Anatomical region } & & \multicolumn{4}{c}{ MNI coordinates } \\
\cline { 2 - 5 } & $k$ & $\mathrm{X}$ & $\mathrm{Y}$ & $\mathrm{Z}$ & \multirow{2}{*}{$t$} \\
\hline Right middle frontal gyrus & 74 & 42 & 54 & 35 & 4.82 \\
& & 50 & 55 & 37 & 4.62 \\
\hline \hline
\end{tabular}

MNI, Montreal Neurological Institute.

Note. Results are thresholded using cluster-level family-wise error corrected $p<.05$, with a cluster extent threshold of $k>20$.

middle frontal gyrus (MFG) (see Figure 3 and Table 2). No significant cluster had emerged in the reverse contrast (i.e., 3 months $>$ baseline and AT $<$ AC). Post hoc correlation analyses among the scanned sample $(N=34)$ revealed that $\mathrm{CT}$ changes in this cluster were significantly associated with changes in RAVLT - total learning $(r=.377 ; p=.028)$, but not digit span forward scores $(r=-.231 ; p=.189)$.

\section{DISCUSSION}

The current study examined the effects of a low-intensity AT intervention on cognitive outcomes and GM CT across 3 months. In the cognitive domains, we observed significant improvements in working memory span and immediate memory among AT participants relative to their AC counterparts. In relation to the AT-associated neuroplasticity, CT in a right MFG cluster was significantly increased among AT participants relative to their AC counterparts. Additionally, the changes in the CT of this right MFG cluster were found to be associated with improvements in immediate memory, alluding to the neuroplastic mechanisms of AT. Overall, these novel results suggest that AT, executed as a low-intensity intervention across 3 months, can engender selective cognitive gains and associated neuroplastic changes, even among individuals with MCI.

Repeated sessions of art production and evaluation provided our AT participants with plenty of opportunities to immerse themselves in a state of flow associated with enhanced focus and sustained engagement while working on their art creations. Furthermore, they frequently integrated multifaceted information to arrive at an artistic judgment (Chilton, 2013). In the process, participants were trained to accommodate multiple pieces of information in their immediate attentional span, so as to readily act on them. Such training would naturally translate to gains on an attentional span test such as the digit span forward. However, similar ATrelated improvements were not observed for the digit span backward test. Despite having similar testing procedures as the digit span forward, the digit span backward additionally involved an executive control component, in the manipulation of information. This additional demand was found to be associated with increased frontoparietal activation (Sun et al., 2005), alluding to the greater recruitment of executive control resources. Such executive control involving the manipulation of information within working memory was unlikely to be practiced upon in our AT; hence, scores on the digit span backward did not appear to benefit from AT.

We have also observed significant AT-associated improvements for immediate memory. Admittedly, we were not expecting AT to have a significant impact on memory outcomes. Nevertheless, AT might have led to the use of various encoding strategies that enhance immediate memory (Frankenmolen, Fasotti, Kessels, \& Oosterman, 2018). This may be attributed to the frequent exposure and processing of art-related stimuli in AT (Smith, 2016), which might have primed the use of imagery-related strategies for other contexts beyond art. Additionally, the enhanced attentional span described earlier, would further facilitate the use of different adaptive strategies (e.g., repetition, imagery) to enhance the encoding and retrieval of information in the immediate memory (Unsworth, 2016). This is especially relevant in the context of aging where executive functions are thought to support memory functions in order to compensate for the declining memory ability (Angel, Fay, Bouazzaoui, \& Isingrini, 2010).

In terms of neuroplasticity, we observed significantly increased CT within the right MFG among AT participants relative to their AC counterparts. Although the MFG could be associated with several neurocognitive functions, our post hoc correlation analyses suggested that this increase in the MFG CT was specific to the improvements in immediate memory. This may appear somewhat puzzling at first since memory-related processing is traditionally thought to recruit upon regions in the limbic system and medial temporal lobe, and on the other hand, working memory/attention was often associated with regions in the PFC (e.g., the MFG). Perhaps, AT might have recruited other working memory/attention regions apart from the MFG, hence the GM changes associated with improvements in attentional span may have been localized in the other regions of the PFC. Unfortunately, due to the small sample size of scanned participants and stringent thresholds used in the whole brain analyses, no such clusters were detected.

On the other hand, the significant association between immediate memory gains and MFG CT changes is not unusual. Functional MRI studies (Kondo et al., 2005; Spreng, Madore, \& Schacter, 2018) have observed that the use of various encoding and retrieval strategies during learning and recall was associated with increased activation in the MFG. Furthermore, another study showed that the use of elaborative encoding strategies during a memory test was significantly associated with GM volume in the MFG (Husa et al., 2017). Hence, if the increased CT in the MFG among AT participants can be explained by the increased use of memory-related strategies, this would further lend support to the conjecture that AT might have improved immediate memory via the use of compensatory encoding and retrieval strategies. Of course, this is speculatory at best, future AT interventions should include the frequency of such strategies used as an intervention outcome and attempt to correlate it with cognitive gains and neuroplastic changes.

Our results present important implications for AT in general. As with other psychotherapies, clients' and 
(A)

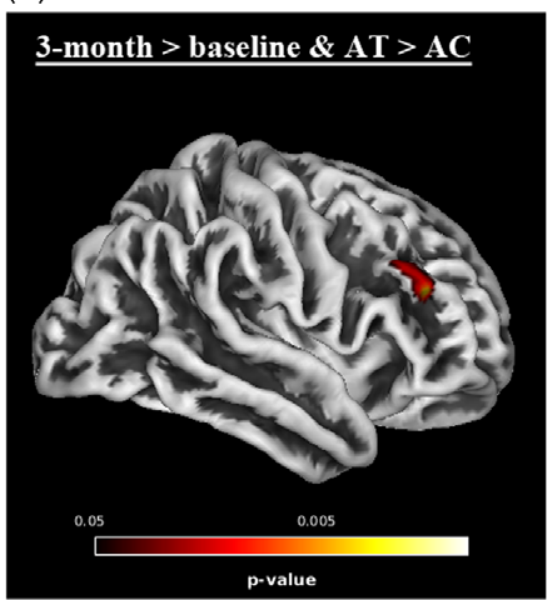

(B)

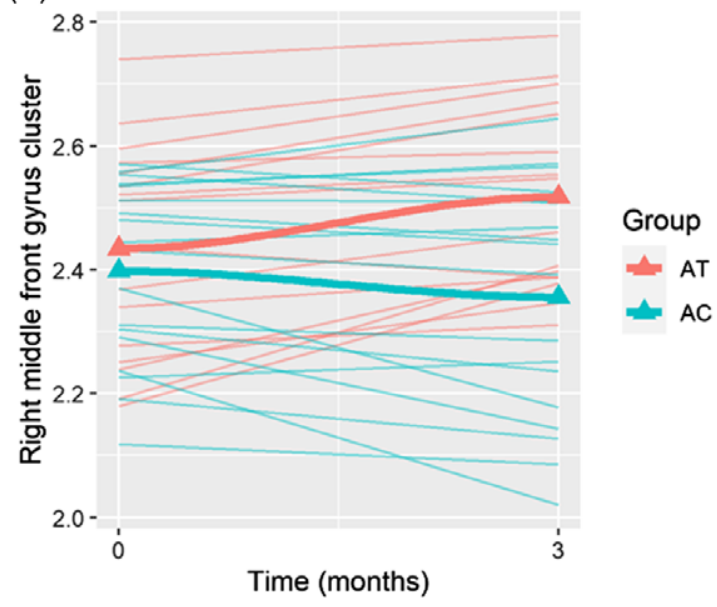

Fig. 3. (A) Significant cluster (FWE-corrected $p<.05$ ) in right middle frontal gyrus within the contrast of 3 months $>$ baseline and AT $>$ AC. (B) trajectories of cortical thickness values $(\mathrm{mm})$ of extracted cluster.

therapists' expectations are major predictors of subsequent treatment outcomes, and such expectations hinge upon on cogent theoretical explanations (Wampold, 2015). Our findings illustrated the neuroplastic mechanism of AT, such neuroscientific explanations, backed by empirically derived brain-behavior relationships, would supplement existing AT-related psychological explanations to instill greater confidence among art therapists and their clients. This will ultimately facilitate the therapeutic process. More importantly, as our results have shown, AT could be used, not just as a complementary treatment, but as a standalone treatment for mild cases of cognitively impaired individuals. In particular, AT would appeal to individuals who are keen on exercising their creativity to augment cognitive functions.

The current study is subjected to some limitations. First, this was an open-label study, in which participants and the experimenters were aware of which treatment group the former was assigned to. There was a small possibility that the results would have been confounded by observerexpectancy effects among experimenters and the differences in participants' treatment expectancies between both groups. Second, although our sample-size calculation indicated an adequate sample size based on previous effect sizes estimates, it appears that the current sample size may have been inadequate to detect weaker treatment-related effects, such as in the block design test. Future larger sampled studies are needed to verify these weaker treatment-related effects. Third, although we had used the block design test to assess visuospatial functions, in actuality, this is not a 'clean' test of visuospatial function. Its scoring took into account of speed, ontop of accuracy, alluding to a significant involvement of processing speed ontop of visuospatial functions. This makes it difficult to interpret the block design test-related results. Future studies may consider including processing speed measures or visuospatial tests in which speed/reaction time is irrelevant, such as the Judgment of Line Orientation test. Fourth, given the exploratory nature of the study and small sample size, we did not carry out corrections for the multiple testing for the cognitive outcomes. Admittedly, the current significant findings would not survive a stringent multiple testing correction. Fifth, although the RAVLT has alternate forms (using different word lists) that could be used for repeated measurements to eliminate practice effects (Hawkins, Dean, \& Pearlson, 2004), we did not use such alternate forms because, unlike the original, the alternate forms have not been adapted to the local context. Sixth, there are concerns that both groups of participants may not be comparable at baseline. For instance, the AT group had higher (though not statistically significant) RAVLT-Total learning scores. This better learning ability meant that the AT participants might stand to reap greater benefit from AT, than if the AC group had been assigned to AT instead. Finally, there were also some limitations relating to the use of the $\mathrm{AC}$ as the control group. Although this $\mathrm{AC}$ was intended to control for confounds relating to social interactions, this AC may inadvertently be associated with other confounds. For instance, AC participants' levels of cognitive functioning could have benefited from their healthier lifestyles-as intended by the didactic sessions they have attended. This could perhaps partially account for the relatively weak AT treatment gains. Furthermore, given the multicomponent nature of the current AT, a comparison to a vastly different treatment condition such as the $\mathrm{AC}$ may not allow one to infer exactly which component of AT was driving the treatment gains.

\section{CONCLUSION}

In conclusion, our AT intervention on participants with MCI has led to significant gains in working memory span and immediate memory, as well as related CT increase in the rMFG. Taken together, AT is a neuroplastic intervention capable of delaying or remediating certain age-related cognitive impairment. Our preliminary findings would 
provide some support for using AT in the context of agerelated cognitive decline. Given the limited sample size of the current study and the paucity of similar studies, future studies should attempt to replicate current findings with a larger sample.

\section{ACKNOWLEDGEMENTS}

This work was supported by Research Donations from Kwan Imm Thong Hood Cho Temple and Lee Kim Tah Holdings Pte Ltd, under the Mind Science Centre, Department of Psychological Medicine, National University of Singapore.

\section{CONFLICTS OF INTEREST}

The authors have nothing to disclose.

\section{SUPPLEMENTARY MATERIAL}

To view supplementary material for this article, please visit https://doi.org/10.1017/S1355617720000697

\section{REFERENCES}

Angel, L., Fay, S., Bouazzaoui, B., \& Isingrini, M. (2010). Individual differences in executive functioning modulate age effects on the ERP correlates of retrieval success. Neuropsychologia, 48(12), 3540-3553. doi: 10.1016/j.neuropsychologia.2010.08.003.

Ashburner, J. (2007). A fast diffeomorphic image registration algorithm. Neuroimage, 38(1), 95-113.

Bolwerk, A., Mack-Andrick, J., Lang, F.R., Dörfler, A., \& Maihöfner, C. (2014). How art changes your brain: differential effects of visual art production and cognitive art evaluation on functional brain connectivity. PLoS ONE, 9(7), 1-8. doi: 10. 1371/journal.pone.0101035.

Chilton, G. (2013). Art therapy and flow: a review of the literature and applications. Art Therapy, 30(2), 64-70. doi: 10.1080/ 07421656.2013 .787211$.

Cohen, G. (2006). Research on creativity and aging: the positive impact of the arts on health and illness. Generations, 30(1), 7-15.

Dahnke, R., Yotter, R.A., \& Gaser, C. (2013). Cortical thickness and central surface estimation. Neuroimage, 65, 336-348.

D'Elia, L., \& Satz, P. (1996). Color trails test. Odessa, FL: Psychological Assessment Resources.

Desikan, R.S., Ségonne, F., Fischl, B., Quinn, B.T., Dickerson, B.C., Blacker, D., ... Hyman, B. T. (2006). An automated labeling system for subdividing the human cerebral cortex on MRI scans into gyral based regions of interest. Neuroimage, 31(3), 968-980.

Fielding, S., Fayers, P., \& Ramsay, C.R. (2012). Analysing randomised controlled trials with missing data: choice of approach affects conclusions. Contemporary Clinical Trials, 33(3), 461-469. doi: 10.1016/j.cct.2011.12.002.

Frankenmolen, N.L., Fasotti, L., Kessels, R.P.C., \& Oosterman, J.M. (2018). The influence of cognitive reserve and age on the use of memory strategies. Experimental Aging Research, 44(2), 117-134.

Hattori, H., Hattori, C., Hokao, C., Mizushima, K., \& Mase, T. (2011). Controlled study on the cognitive and psychological effect of coloring and drawing in mild Alzheimer's disease patients. Geriatrics and Gerontology International, 11(4), 431437. doi: 10.1111/j.1447-0594.2011.00698.x.

Hawkins, K.A., Dean, D., \& Pearlson, G.D. (2004). Alternative forms of the rey auditory verbal learning test: a review. Behavioural Neurology, 15, 940191. doi: 10.1155/2004/940191.

Husa, R.A., Gordon, B.A., Cochran, M.M., Bertolin, M., Bond, D.N., \& Kirchhoff, B.A. (2017). Left caudal middle frontal gray matter volume mediates the effect of age on self-initiated elaborative encoding strategies. Neuropsychologia, 106, 341-349. doi: 10.1016/j.neuropsychologia.2017.10.004.

Klainin-Yobas, P., Kowitlawakul, Y., Lopez, V., Tang, C.T., Hoek, K.E., Gan, G.L., . . Mahendran, R. (2019). The effects of mindfulness and health education programs on the emotional state and cognitive function of elderly individuals with mild cognitive impairment: a randomized controlled trial. Journal of Clinical Neuroscience, 68, 211-217. doi: 10.1016/j.jocn.2019. 05.031.

Kondo, Y., Suzuki, M., Mugikura, S., Abe, N., Takahashi, S., Iijima, T., \& Fujii, T. (2005). Changes in brain activation associated with use of a memory strategy: a functional MRI study. Neurolmage, 24(4), 1154-1163. doi: 10.1016/j.neuroimage.2004.10.033.

Kontson, K., Megjhani, M., Brantley, J., Cruz-Garza, J., Nakagome, S., Robleto, D., ... Contreras-Vidal, J. (2015). 'Your brain on art': emergent cortical dynamics during aesthetic experiences. Frontiers in Human Neuroscience, 9, 626. Retrieved from https://www.frontiersin.org/article/10.3389/fnhum.2015.00626

Lampit, A., Hallock, H., \& Valenzuela, M. (2014). Computerized cognitive training in cognitively healthy older adults: a systematic review and meta-analysis of effect modifiers. PLOS Medicine, 11(11), e1001756.

Lee, C.K.Y., Collinson, S.L., Feng, L., \& Ng, T.-P. (2012). Preliminary normative neuropsychological data for an elderly Chinese population. The Clinical Neuropsychologist, 26(2), 321-334. doi: 10.1080/13854046.2011.652180.

Lee, R., Wong, J., Lit Shoon, W., Gandhi, M., Lei, F., EH, K., ... Mahendran, R. (2019). Art therapy for the prevention of cognitive decline. Arts in Psychotherapy, 64(April 2018), 20-25. doi: 10. 1016/j.aip.2018.12.003.

Lusebrink, V.B. (2004). Art therapy and the brain: an attempt to understand the underlying processes of art expression in therapy. Art Therapy, 21(3), 125-135. doi: 10.1080/07421656.2004. 10129496.

Maujean, A., Pepping, C.A., Kendall, E., Maujean, A., Pepping, C.A., \& Kendall, E. (2014). A systematic review of randomized controlled studies of art therapy. Art Therapy, 31(1), 37-44. doi: 10.1080/07421656.2014.873696.

Petersen, R.C. (2004). Mild cognitive impairment as a diagnostic entity. Journal of Internal Medicine, 256(3), 183-194. doi: 10. 1111/j.1365-2796.2004.01388.x.

Petersen, R.C., Caracciolo, B., Brayne, C., Gauthier, S., Jelic, V., \& Fratiglioni, L. (2014). Mild cognitive impairment: a concept in evolution. Journal of Internal Medicine, 275(3), 214-228. doi: 10.1111/joim.12190.

Pike, A.A. (2013). The effect of art therapy on cognitive performance among ethnically diverse older adults. Art Therapy, 30(4), 159-168. doi: 10.1080/07421656.2014.847049.

Rey, A. (1941). L'examen psychologique dans les cas d'encéphalopathie traumatique. (Les problems.). Archives de Psychologie.

Rodakowski, J., Saghafi, E., Butters, M.A., \& Skidmore, E.R. (2015). Non-pharmacological interventions for adults with 
mild cognitive impairment and early stage dementia: an updated scoping review. Molecular Aspects of Medicine, 43-44, 38-53. doi: 10.1016/j.mam.2015.06.003.

Rusted, J., Sheppard, L., \& Waller, D. (2006). A multi-centre randomized control group trial on the use of art therapy for older people with dementia. Group Analysis, 39(4), 517-536. doi: 10. 1177/0533316406071447.

Sherman, D.S., Mauser, J., Nuno, M., \& Sherzai, D. (2017). The efficacy of cognitive intervention in mild cognitive impairment (MCI): a meta-analysis of outcomes on neuropsychological measures. Neuropsychology Review, 27(4), 440-484. doi: 10.1007/s11065017-9363-3.

Smith, A. (2016). A literature review of the therapeutic mechanisms of art therapy for veterans with post-traumatic stress disorder. International Journal of Art Therapy, 21(2), 66-74. doi: 10. 1080/17454832.2016.1170055.

Spreng, R.N., Madore, K.P., \& Schacter, D.L. (2018). Better imagined: neural correlates of the episodic simulation boost to prospective memory performance. Neuropsychologia, 113, 22-28. doi: 10.1016/j.neuropsychologia.2018.03.025.

Sun, X., Zhang, X., Chen, X., Zhang, P., Bao, M., Zhang, D., ... $\mathrm{Hu}, \mathrm{X}$. (2005). Age-dependent brain activation during forward and backward digit recall revealed by fMRI. NeuroImage, 26(1), 36-47. doi: 10.1016/j.neuroimage.2005.01.022.

Till, C., Kuni, B., De Somma, E., Yeh, E.A., \& Banwell, B. (2019). A feasibility study of working memory training for individuals with paediatric-onset multiple sclerosis. Neuropsychological Rehabilitation, 29(8), 1177-1192. doi: 10.1080/09602011.2017. 1372786.

Törnbom, K., \& Danielsson, A. (2018). Experiences of treadmill walking with non-immersive virtual reality after stroke or acquired brain injury - A qualitative study. PLOS ONE, 13(12), e0209214. Retrieved from https://doi.org/10.1371/journal.pone. 0209214.

Ulman, E. (2001). Art therapy: problems of definition. American Journal of Art Therapy, 40(1), 16.

Unsworth, N. (2016). Working memory capacity and recall from long-term memory: examining the influences of encoding strategies, study time allocation, search efficiency, and monitoring abilities. Journal of Experimental Psychology: Learning, Memory, and Cognition, 42, 50-61. doi: 10.1037/xlm0000148.

Wampold, B.E. (2015). How important are the common factors in psychotherapy? an update. World Psychiatry, 14(3), 270-277. doi: 10.1002/wps.20238.

Wechsler, D. (1997). WAIS-III: Administration and scoring manual: Wechsler adult intelligence scale. Psychological Corporation.

Yotter, Rachel A., Thompson, P.M., \& Gaser, C. (2011). Algorithms to improve the reparameterization of spherical mappings of brain surface meshes. Journal of Neuroimaging, 21(2), e134-e147.

Yotter, Rachel, A., Dahnke, R., Thompson, P.M., \& Gaser, C. (2011). Topological correction of brain surface meshes using spherical harmonics. Human Brain Mapping, 32(7), 1109-1124. 